\title{
Highly accurate nuclear and electronic stopping cross sections derived using Monte Carlo simulations to reproduce measured range data
}

\author{
Klaus Wittmaack ${ }^{1}$ and Andreas Mutzke ${ }^{2}$ \\ ${ }^{1}$ Helmholtz Zentrum München, Institute für Strahlenschutz, D-85758 Neuherberg, Germany \\ ${ }^{2}$ Max-Planck-Institut für Plasmaphysik, EURATOM Association, Teilinstitut Greifswald, \\ D-17491 Greifswald, Germany
}

(Received ...)

We have examined and confirmed the previously unexplored concept of using measured projected ranges of ions implanted in solids to derive a quantitative description of nuclear interaction and electronic stopping. The idea was to perform Monte Carlo calculations of range distributions and to search for those input parameters that generate, over a wide band of energies, the best possible agreement with accurate experimental range data. The projectile-target combination studied was ${ }^{11} \mathrm{~B}$ in $\mathrm{Si}$, in which case 98 data contained in 12 sets reported by 10 different groups were compiled between $1 \mathrm{keV}$ and $8 \mathrm{MeV}$. Detailed examination revealed set-wise systematic errors up to $\pm 8 \%$. Their removal resulted in a refined data base with 93 ranges featuring only statistical uncertainties (mean standard deviation 1.8\%; five outliers not considered any further). Ultimately, the Monte Carlo calculations reproduced the 93 refined ranges very well, with a mean ratio of $1.002 \pm 1.7 \%$. The input parameters required to achieve this very high level of agreement were identified to be as follows. Nuclear interaction is best described by the $\mathrm{Kr}-\mathrm{C}$ potential, but only when used in obligatory combination with the Lindhard-Scharff (LS) screening length. Up to $300 \mathrm{keV}$ the electronic stopping cross section is proportional to the projectile velocity, i.e., LS-type, $S_{\mathrm{e}}=k S_{\mathrm{e}, \mathrm{LS}}$, with $k=1.46 \pm 0.01$. At higher energies $S_{\mathrm{e}}$ falls progressively short of $k S_{\mathrm{e}, \mathrm{LS}}$. Around the Bragg peak, i.e., between 0.8 and $10 \mathrm{MeV}, S_{\mathrm{e}}$ is described by an adjustable function with fit parameters selected to tailor the peak shape properly (flat-topped region between 1.5 and $5 \mathrm{MeV}$ ). The reliability of our results is confirmed by showing that calculated and measured isotope effects for ranges of ${ }^{10} \mathrm{~B}$ and ${ }^{11} \mathrm{~B}$ in Si agree within experimental uncertainty $( \pm 0.25 \%)$. Furthermore, the range-based $S_{\mathrm{e}, \mathrm{R}}(E)$ reported here predicts the scarce experimental data derived from energy loss in projectile transmission through thin Si foils to within $2 \%$ or better. By contrast, $S_{\mathrm{e}}(E)$ data available from different types of stopping power tables must be rated inaccurate, the deviations from $S_{\mathrm{e}, \mathrm{R}}(E)$ ranging between $-40 \%$ and $+14 \%$.

DOI: PACS numbers: 79.20.Rf, 34.35.+a, 34.50.Bw, 61.72.sh 


\section{INTRODUCTION}

Recent studies on the slowing-down of energetic ions in matter have addressed a wide variety of topics. Arranged roughly in the order of increasing projectile energy or velocity, noteworthy examples include the following: the correlation between electronic stopping and ion induced electron emission $[1,2,3]$, the role of $s$ and $d$ electrons in electronic stopping of slow protons and deuterons $[1,4,5,6,7]$, systematic differences in electronic stopping of low-energy $\mathrm{H}^{+}$and $\mathrm{He}^{+}$ions $[6,8]$, the importance of exact knowledge of nuclear stopping (screening length) in low-energy ion scattering [9], the applicability of the reciprocity approach [10] for predicting ranges of slow heavy ions in compounds $[11,12,13]$, modelling of range distributions in crystalline silicon [14], and measurements and interpretation of electronic stopping of low- and medium-mass ions in solids at energies around the Bragg peak and below [15,16,17]. However, reasonably accurate knowledge of electronic stopping cross sections $S_{\mathrm{e}}$ is still available merely for a very limited number of projectile-target combinations, often within narrow ranges of energy.

The standard approach to determining $S_{\mathrm{e}}$ is to measure the amount of energy lost by energetic ions on passage through thin foils $[18,19]$ or gas cells [20]. Issues in such studies are the adequate extraction of the relevant quantity from the measured loss spectrum (mean versus most probable loss), the homogeneity in composition and the uniformity in thickness of the foil, and proper consideration of contributions due to nuclear stopping [21]. Quite generally, the method is reasonably accurate only at energies well above the maximum of the nuclear stopping cross section $S_{\mathrm{n}}$. This is the main reason for the scarcity of data on electronic stopping of heavy ions in medium-mass and heavy-atom targets at energies below the Bragg peak. The stopping and range tables contained in the popular library 'The Stopping and Range of Ions in Matter' (SRIM) [22] constitute an attempt to construct the desired information from data available for low-mass projectiles and targets [23]. Regrettably, the stopping data delivered by SRIM are derived on the basis of the incorrect assumption that theoretical concepts valid for describing electronic stopping at energies above the Bragg peak can be extrapolated down to the lowest energies of interest. The predicted data are often in error by as much as a factor of two, if not more [23].

Given this situation one may wonder whether there are alternative ways to determine stopping cross sections, preferably with free choice of projectile and (solid) target material and basically with no restrictions in terms of energy. The purpose of this study was to examine a universally applicable approach that has not been considered in any detail before. The idea is to first collect measured range data for the chosen projectile-target combination, covering as many orders of magnitude in energy as 
possible. This set of data then serves as the basis for adjusting and refining the input parameters of Monte Carlo calculations performed to calculate range distributions. The approach described here differs completely from the commonly applied method of comparing measured range data with the predictions of SRIM. The intention is not to evaluate the level of agreement or disagreement with calculations involving questionable or poorly defined input parameters but rather to determine the contributions of nuclear interaction and electronic stopping to the observed ranges with an accuracy of $3 \%$ or better.

\section{BASIC ASPECTS}

Given the ambitious goal of this study it is very important to understand how the ranges of implanted ions respond to changes in nuclear and electronic stopping. Quite generally one must take into account that ranges contain integral information on stopping, as opposed to differential energy losses determined by beam transmission through a foil. To derive reasonably accurate knowledge on nuclear stopping it is necessary to have access to data on mean ranges down to few nanometers. The projectile-target combination discussed in this work is B implanted in $\mathrm{Si}$, the main reason being that useful range data are available for wide region of energies, from $1 \mathrm{keV}$ to $8 \mathrm{MeV}$. Furthermore, several sets of results have been reported for both ${ }^{10} \mathrm{~B}$ and ${ }^{11} \mathrm{~B}$ in $\mathrm{Si}$, so that the presence or absence of isotope effects in ranges can be used to check the validity of results obtained with the major isotope alone.

For an overview on the energy dependence of nuclear and electronic stopping Fig. 1 shows $S_{\mathrm{n}}$ (open circles), $S_{\mathrm{e}}$ (solid triangles) and the sum $S_{\mathrm{n}}+S_{\mathrm{e}}$ (gray line) according to the data tables of SRIM. Even though the accuracy is not satisfactory [23], the data serve well to discuss general trends. $S_{\mathrm{n}}$ is proportional to the mean energy $\langle\Delta E\rangle$ lost by the projectile (energy $E$, mass $M_{1}$, atomic number $Z_{1}$ ) in elastic collisions with target atoms $\left(M_{2}, Z_{2}\right)$. Normalized to passage through one nominal monolayer we have $S_{\mathrm{n}}=\langle\Delta E\rangle / n^{2 / 3}$, where $n$ is the number density of target atoms. According to theoretical predictions of Lindhard and Scharff (LS) [24], $S_{\mathrm{e}}$ is commonly assumed to be proportional to the projectile velocity $v$, as long as $v<v_{0} Z_{1}^{2 / 3}$ (Bohr velocity $v_{0}$ ), or $E / M_{1}<25 Z_{1}^{4 / 3} \mathrm{keV} / \mathrm{u}$. Absolute differences between experiment and theory are accounted for by a correction factor $k$, i.e., $S_{\mathrm{e}}=k S_{\text {e,LS}}$. SRIM basically proceeds along this line, but frequently with unexplained, rather strange modifications of $k S_{\text {e,LS }}$ [23]. An example of moderate magnitude is the hump seen in Fig. 1 at energies around $50 \mathrm{keV}$. 
For $\mathrm{H}^{+}, \mathrm{D}^{+}$, and $\mathrm{He}^{+}$incident on metals, velocity-proportional electronic stopping has been observed down to velocities as low as $0.1 v_{0}$, often with a change in gradient at about $0.2 v_{0}\left(E / M_{1}=1\right.$ $\mathrm{keV} / \mathrm{u}$ ), attributed to the onset of stopping due to $d$ electrons [1,4-7]. For B in Si similar threshold effects have not (yet) been reported. Nevertheless, the consequences of such a 'break' in $S_{\mathrm{e}}(v)$ deserve attention. A conceivable velocity dependent form of $S_{\mathrm{e}}$, with a change in slope at $0.2 v_{0}\left(11 \mathrm{keV}{ }^{11} \mathrm{~B}\right)$, is shown in Fig. 1 as a dashed line, labeled $S_{\mathrm{e}, \mathrm{k}}$.

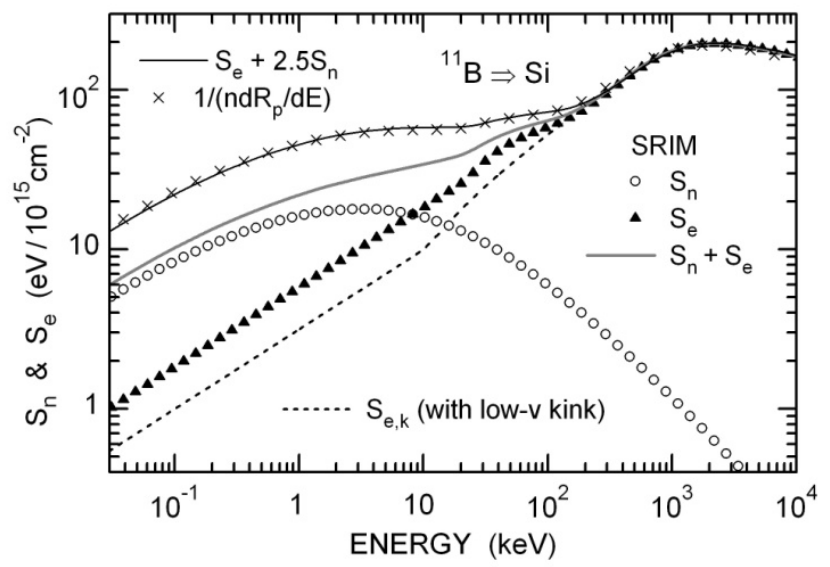

FIG. 1. Energy dependent nuclear and electronic stopping of ${ }^{11} \mathrm{~B}$ in Si (SRIM, version 2006.02). The short-dashed line represents the hypothetical case of two-step velocity-proportional stopping with a kink at $11 \mathrm{keV}$.

Going up in energy, towards and beyond the upper limit of velocity-proportional electronic stopping, we are dealing with partially stripped ions. Optimum theoretical treatment of the relevant phenomena is an ongoing challenge [25]. Turning to the low-energy end of the data in Fig. 1, i.e. below $1 \mathrm{keV}, S_{\mathrm{n}}$ exceeds $S_{\mathrm{e}}$ by up to a factor of about five. Interestingly, the ratio $S_{\mathrm{n}} / S_{\mathrm{e}}$ becomes roughly constant at very low energies, $E<0.1 \mathrm{keV}$. Hence, even when measured at very low energies, ranges must be expected to contain a sizable contribution due to electronic stopping.

With stopping cross sections at hand, one may estimate ranges using simplified theoretical concepts. According to the continuous slowing-down approximation $[25,26]$, the total range $R$ may be written

$$
R=\int_{E}^{0} \frac{\mathrm{d} E^{\prime}}{n\left(S_{\mathrm{n}}\left(E^{\prime}\right)+S_{\mathrm{e}}\left(E^{\prime}\right)\right)} .
$$

Ranges thus obtained numerically with the stopping data of Fig. 1 are presented in Fig. 2. The simplest case is the range $R_{\mathrm{e}}=R\left(S_{\mathrm{e}}\right)$ calculated in the absence of nuclear stopping, open triangles in Fig. 2. Such data are of relevance in studies on ranges in single crystals under channeling conditions 
[14]. Taking $S_{\mathrm{n}}$ into account, the total range $R_{\mathrm{n}, \mathrm{e}}=R\left(S_{\mathrm{n}}+S_{\mathrm{e}}\right)$ in amorphous material drops by a factor of about six at the lowest energies (gray line). With increasing energy $R_{\mathrm{n}, \mathrm{e}}$ approaches $R_{\mathrm{e}}$, but even at about $600-700 \mathrm{keV}$, where $S_{\mathrm{n}}$ amounts to only $1 \%$ of $S_{\mathrm{n}}+S_{\mathrm{e}}$, the total range is $15 \%$ lower then $R_{\mathrm{e}}$. Hence it is not at all justified to ignore the contribution of nuclear stopping to the range at 'high' energies at which electronic stopping strongly dominates [25]. As to the ability of detecting deviations of $S_{\mathrm{e}}$ from an initially assumed energy (velocity) dependence, the $45 \%$ difference between $S_{\mathrm{e}}$ and the hypothetical case $S_{\mathrm{e}, \mathrm{k}}$ (Fig. 1) gives rise to a detectable 8\%-difference in range below the kink at 11 $\mathrm{keV}$, dashed line in Fig. 2. The difference increases to as much as $27 \%$ between 40 and $60 \mathrm{keV}$, easy to identify in range data of proper quality.

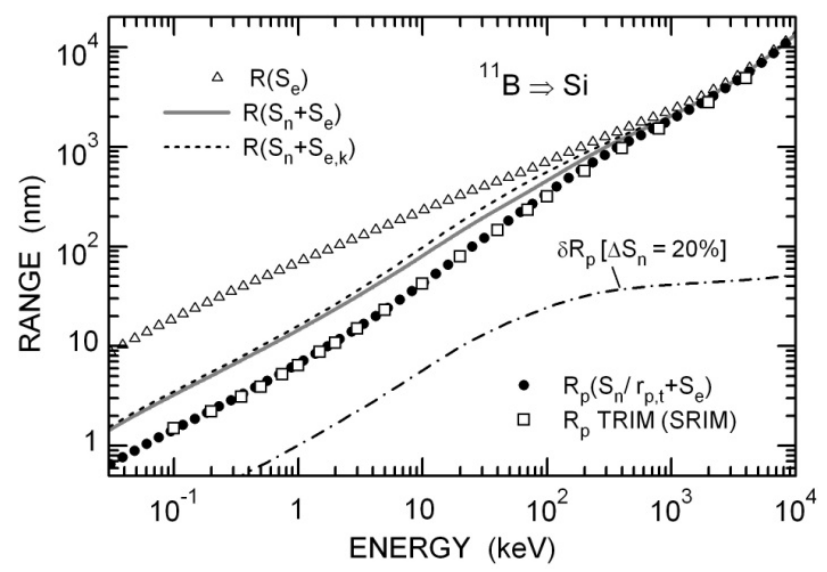

FIG. 2. Total and projected ranges of ${ }^{11} \mathrm{~B}$ in $\mathrm{Si}$ estimated using a simple analytical approach in combination with the stopping cross sections of Fig. 1. For details see text.

Up to now the discussion has been of limited relevance because only total ranges were considered. To estimate measured projected ranges, the assumption was made that the effect of scattering in elastic collisions can be simulated by enlarging $S_{\mathrm{n}}$ to become $S_{\mathrm{n}} / r_{\mathrm{p}, \mathrm{t}}$, where $r_{\mathrm{p}, \mathrm{t}}=R_{\mathrm{p}} / R$ is the projection factor. For ${ }^{11} \mathrm{~B}$ in Si this $M_{2} / M_{1}$ dependent factor is $r_{\mathrm{p}, \mathrm{t}}=0.4 \pm 0.02$ according to range studies [27], in good agreement with theoretical predictions [28]. The projected ranges thus obtained are shown in Fig. 2 as small solid circles. They agree remarkably well with the open squares which represent the mean projected ranges derived from range distributions calculated using the Monte Carlo code TRIM (TRansport of Ions in Matter) contained in SRIM as a separate package (here referred to as TRIMsrim). This good agreement suggests that one can identify changes in projected range that may arise in case that the true nuclear stopping cross section $S_{\mathrm{n}}$ differs from the initially assumed value $S_{\mathrm{n}, \mathrm{i}}$ by a small factor $b, S_{\mathrm{n}}=b S_{\mathrm{n}, \mathrm{i}}$. A reasonable example is $b=1.20$. The ratio $R_{\mathrm{p}}\left(S_{\mathrm{n}, \mathrm{i}}\right) / R_{\mathrm{p}}\left(S_{\mathrm{n}}\right)$ turned out to be 1.185 at the lowest energies, decreasing slowly to 1.10 at $60 \mathrm{keV}$, still 
amounting to 1.03 at $600-700 \mathrm{keV}$. The dash-dotted line in Fig. 2 shows the difference $\delta R_{\mathrm{p}}=R_{\mathrm{p}}\left(S_{\mathrm{n}, \mathrm{i}}\right)$ - $R_{\mathrm{p}}\left(S_{\mathrm{n}}\right)$. We conclude that up to energies at which $S_{\mathrm{e}}$ exceeds $S_{\mathrm{n}}$ by two orders of magnitude the total as well as the projected ranges respond to the finer details of $S_{\mathrm{n}}$ in a detectable manner.

Inspecting Eq. (1) again one will note that, in principle, the total stopping cross may be determined as the inverse derivative $1 / n(\mathrm{~d} R / \mathrm{d} E)$, an approach briefly discussed before [25], with proper caution concerning nuclear scattering and stopping. As an example, the crosses in Fig. 1 show results obtained by applying this method to the $R_{\mathrm{p}}$-data of Fig. 2 . As it should be, the calculated data agree with the sum $S_{\mathrm{e}}+S_{\mathrm{n}} / r_{\mathrm{p}, \mathrm{t}}=S_{\mathrm{e}}+2.5 S_{\mathrm{n}}$ that was used to determine $R_{\mathrm{p}}$ (thin black line). At first sight this result may be viewed merely a test of internal consistency. The important point, however, is that even if the number and spacing of the range data would allow the derivative to be obtained with adequate accuracy, it is impossible without further information to separate the sum into the two contributions of interest, $S_{\mathrm{e}}$ and $S_{\mathrm{n}}$. To achieve the goal one needs to use the procedure discussed below.

\section{DETAILED CONSIDERATIONS}

\section{A. Nuclear stopping}

Even though Fig. 2 is very useful in that the results provide means to estimate the effect of changes in $S_{\mathrm{e}}$ and $S_{\mathrm{n}}$ on ranges, the relevant formula, Eq. (1), does not properly reflect the actual procedure used in range calculations. Whereas projectile interaction with target electrons is usually considered to give rise to a uniform, non-local force that causes continuous slowing down, so that $S_{\mathrm{e}}$ is a suitable input parameter, an equivalent nuclear stopping cross section is not applicable. The relevant quantity for calculating the elastic (nuclear) energy transfer and scattering in collisions between two atoms (interatomic spacing $r$, elementary charge e) is the projectile-target interaction potential. Screened Coulomb potentials $V(r, a)=\left(Z_{1} Z_{2} \mathrm{e}^{2} / r\right) \phi(r / a)$ commonly serve the purpose. Much effort has been devoted to finding appropriate screening functions $\phi(r / a)$. Most frequently used are Molière's analytical description $\phi_{\mathrm{M}}[29]$ of the Thomas-Fermi function, the $\mathrm{Kr}$-C function $\phi_{\mathrm{KrC}}$ [26], and the screening function $\phi_{\text {univ }}$ of the universal potential [30]. The dependence of $\phi(r / a)$ on the atomic numbers $Z_{1}$ and $Z_{2}$ is buried in the screening length $a$ which is of the general form

$$
a=\left(\frac{9 \pi^{2}}{128}\right)^{1 / 3} \frac{\mathrm{a}_{0}}{Z_{1,2}^{*}}=0.8853 \frac{\mathrm{a}_{0}}{Z_{1,2}^{*}}=\frac{a^{*}}{Z_{1,2}^{*}}
$$


with $a^{*}=0.4685 \times 10^{-8} \mathrm{~cm}$. Depending on the choice of $Z_{1,2}^{*}$, three variants of the parameter $a$ must be distinguished [25], $a_{\mathrm{LS}}=a^{*} /\left(Z_{1}^{2 / 3}+Z_{2}^{2 / 3}\right)^{1 / 2}[24], a_{\mathrm{Fir}}=a^{*} /\left(Z_{1}^{1 / 2}+Z_{2}^{1 / 2}\right)^{2 / 3}[31]$, and $a_{\mathrm{ZB}}=a^{*} /\left(Z_{1}^{0.23}+Z_{2}^{0.23}\right)$ [30]. Inspection of the literature leads to the conclusion that $\phi(r / a)$ and $a$ may be combined deliberately. Examples can be found in the work of Kalbitzer and Oetzmann [32] and Wilson et al. [26] who examined $\phi_{\mathrm{M}}(r / a)$ [29] in combination with $a_{\mathrm{LS}}$ and $a_{\text {Fir }}$, respectively, even though Molière used the definition $a=a_{\mathrm{TF}}=a^{*} / Z_{2}^{1 / 3}$, the Thomas-Fermi length unit. The association of $a_{\mathrm{LS}}$ with $a_{\mathrm{TF}}$ was mentioned by Lindhard and Scharff [24].
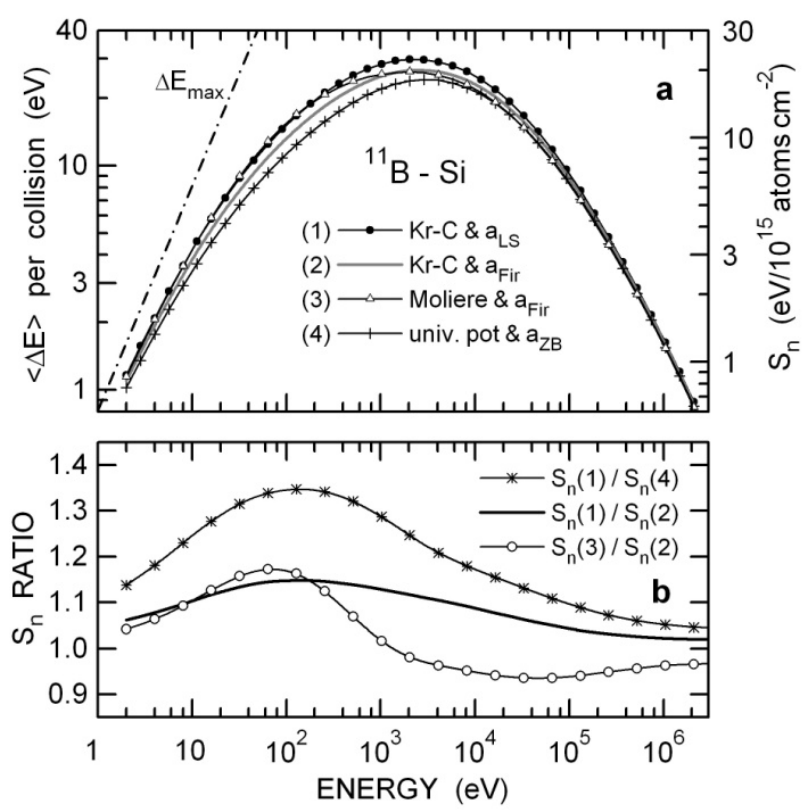

FIG. 3. (a) Mean energy loss per collision (left-hand scale) and nuclear stopping cross section (right-hand scale) for impact of ${ }^{11} \mathrm{~B}$ on Si versus the projectile energy. Data are shown for three different screening functions and three different screening lengths. (b) Ratios of the stopping cross sections in panel (a).

We have determined mean energy losses $\langle\Delta E\rangle$ in elastic binary collisions of ${ }^{11} \mathrm{~B}$ with Si for four combinations of $\phi(r / a)$ with $a$, see Fig. 3(a). The calculations were performed using the Monte Carlo code SDTrimSP [33], version 5.08, the current multi-purpose version of the program TRIM originally developed by Biersack and Haggmark [34]. SDTrimSP can handle both 'static' targets (S), renewed after every impact, or 'dynamically' altered targets (D) with a composition changing due to retained projectiles or bombardment induced mixing. Various aspects of sputtering (SP) may also be studied [35]. Here only the static code is of interest, but improvements in numerical procedures implemented in course of time were taken advantage of [35]. 
The results shown in Fig. 3(a) have in common that $\langle\Delta E\rangle$ and $S_{\mathrm{n}}=\langle\Delta E\rangle / n^{2 / 3}\left(\mathrm{Si}: n^{2 / 3}=1.353 \times 10^{15}\right.$ $\mathrm{cm}^{-2}$ ) increase with increasing projectile energy (initially roughly as $E^{0.75}$ ), pass through a maximum located between 2 and $4 \mathrm{keV}$ and then decrease, finally as $E^{-0.8}$. At very low energies, $\langle\Delta E\rangle$ approaches the maximum energy transfer $\Delta E_{\max }$ achievable in head-on collisions (dash-dotted line). Of key importance here are the differences in $\langle\Delta E\rangle$ observed with the different combinations of $\phi(r / a)$ and $a$. To a limited extent their effect may be separated by considering ratios of $\langle\Delta E\rangle$ or $S_{\mathrm{n}}$, see Fig. 3(b). As a first example we keep $\phi$ fixed, $\phi=\phi_{\mathrm{KrC}}$, to vary $a$. The $S_{\mathrm{n}}$-ratio for $\phi_{\mathrm{KrC}}\left(r / a_{\mathrm{LS}}\right)$ versus $\phi_{\mathrm{KrC}}\left(r / a_{\mathrm{Fir}}\right)$, thick black line labeled $S_{\mathrm{n}}(1) / S_{\mathrm{n}}(2)$, exhibits a maximum of 1.15 at $\sim 140 \mathrm{eV}$ compared to $a_{\mathrm{Fir}} / a_{\mathrm{LS}}=1.115$. Hence, apart from the fact that all $S_{\mathrm{n}}$-ratios are energy dependent, even the maximum value is not directly related to the $a$-ratio. Next, for two different screening functions, $\phi_{\mathrm{KrC}}$ and $\phi_{\mathrm{M}}$, with the same $a=a_{\text {Fir }}$, the $S_{\mathrm{n}}$-ratio exhibits an oscillatory character with a maximum of 1.17 and a minimum of 0.93, thin line through open circles in Fig. 3(b), $S_{\mathrm{n}}(3) / S_{\mathrm{n}}(2)$. The consequences of this behavior for the ratio of the respective ranges are impossible to predict. The situation appears to be much simpler in the third case studied, $\phi_{\mathrm{Kr} C}\left(r / a_{\mathrm{LS}}\right)$ versus $\phi_{\mathrm{univ}}\left(r / a_{\mathrm{ZB}}\right)$, line through asterisks, $S_{\mathrm{n}}(1) / S_{\mathrm{n}}(4)$, with a maximum $S_{\mathrm{n}}$-ratio as large as 1.35 . In view of this result we expect the range calculated with $\phi_{\text {univ }}\left(r / a_{\mathrm{Z}}\right)$ to be much larger than for $\phi_{\mathrm{KrC}}\left(r / a_{\mathrm{LS}}\right)$, notably below $10 \mathrm{keV}$.

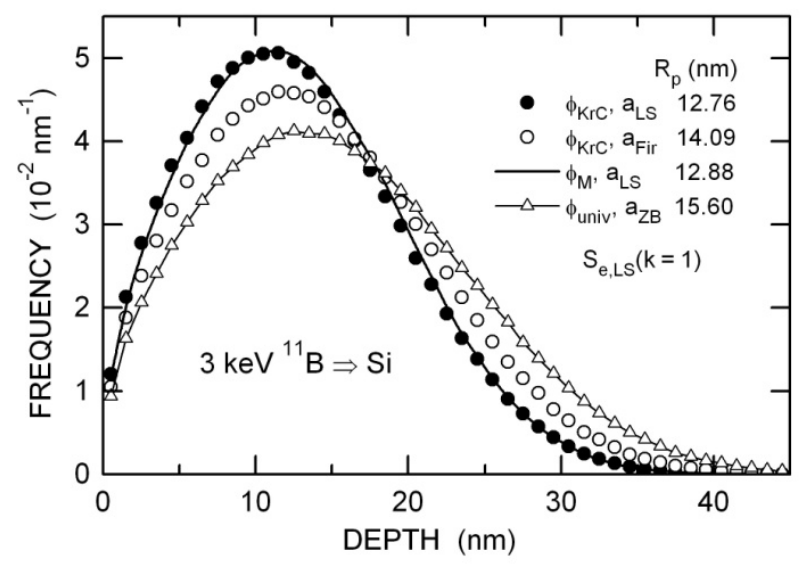

FIG. 4. Range distribution of $3 \mathrm{keV} \mathrm{B}$ in Si calculated with various combinations of the screening function and the screening length.

Examples of range distribution calculated with SDTrimSP for $3 \mathrm{keV}{ }^{11} \mathrm{~B}$ (LS electronic stopping, $k=1)$ are presented in Fig. 4. Quite generally, the data confirm expectation in that the choice of $\phi(r / a)$ and $a$ affects shape, height and position of the distributions quite strongly. As specified in Fig. 4 , the mean projected ranges $R_{\mathrm{p}}$ extend from a minimum of $12.76 \mathrm{~nm}$ for $\phi_{\mathrm{KrC}}\left(r / a_{\mathrm{LS}}\right)$ to a maximum of 
$15.60 \mathrm{~nm}$ for $\phi_{\mathrm{univ}}\left(r / a_{\mathrm{ZB}}\right)$, a difference by $22.3 \%$. On the other hand, the range distributions derived with $\phi_{\mathrm{KrC}}\left(r / a_{\mathrm{LS}}\right)$ and $\phi_{\mathrm{M}}\left(r / a_{\mathrm{LS}}\right)$ are seen to be very similar, solid circles vs straight line. This is a consequence of the fact that, between 1 and $3 \mathrm{keV}$, the mean energy loss per collision is essentially the same for theses two screening functions, see Fig. 3(a), provided one uses the same screening length. Summing up, the data in Figs. 3 and 4 suggest that, with an accurate set of experimental data at hand, it should not be too difficult to identify the combination of screening function and screening length that is best suited to describe nuclear interaction in slowing-down of ${ }^{11} \mathrm{~B}$ implanted in Si.

A brief remark is in place concerning the large amount of range data reported in the literature on the basis of TRIMsrim calculations. An important question is how well results agree when obtained by TRIMsrim or SDTrimSP. To our knowledge this issue has not been addressed before. For a meaningful comparison the fixed input parameters of TRIMsrim ( $\left.\phi=\phi_{\text {univ }}, a=a_{\mathrm{ZB}}\right)$ had to be adopted in the SDTrimSP calculations. At reduced energies $E / M_{1}<2 \mathrm{keV} / \mathrm{u}$, SRIM always assumes LS electronic stopping [23], with $k=1.4$ for B in Si. Range distributions calculated for 5 and $20 \mathrm{keV}^{11} \mathrm{~B}$ in Si are compared in Fig. 5. It is evident at first glance that, at the same implantation energy, the distributions are essentially indistinguishable. This agreement is confirmed examining the three most

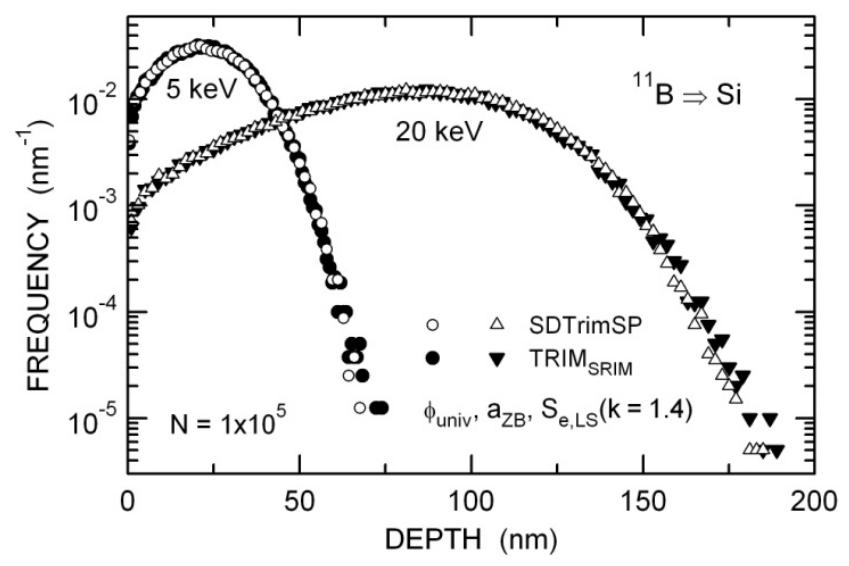

FIG. 5. Range distributions of B in Si calculated with two versions of the Monte Carlo code TRIM, using the same input parameters for nuclear interaction and electronic stopping. To improve readability, every second data point of SDTrimSP was skipped at $5 \mathrm{keV}$.

important integral quantities derived from the distributions, the retained fraction (TRIMsrim vs SDTrimSP; $5 \mathrm{keV}: 0.955$ vs $0.951 \bullet 20 \mathrm{keV}: 0.981$ vs 0.981$)$, the mean projected range $R_{\mathrm{p}}[\mathrm{nm}](22.9$ vs $22.8 \bullet 79.3$ vs 79.7$)$ and the range straggling $\Delta R_{\mathrm{p}}[\mathrm{nm}](11.4$ vs $11.4 \bullet 31.5$ vs 31.7$)$. On average, the numbers denoting the same quantity differ by less than $\pm 0.5 \%$ (for $N=1 \times 10^{5}$ ). We conclude that 
any difference in range data that may have been observed using either TRIMsrim or SDTrimSP must be attributed to differences in the chosen stopping parameters.

\section{B. Electronic stopping}

As pointed out before, range distributions contain integral information on nuclear and electronic energy losses. Hence, at energies at which electronic stopping dominates the distributions should somehow mirror details of the velocity dependence $S_{\mathrm{e}}(v)$. This supposition was examined by using

SDTrimSP to calculate range distributions for $300 \mathrm{keV}^{11} \mathrm{~B}$ in Si, assuming $S_{\mathrm{e}}(v) \propto E^{q}$, the exponent $q$ being varied between 0.4 and 0.6. Results for the two extreme cases are presented in Fig. 6. The effect of increasing $q$ is to cause a distinct broadening of the range distribution and a corresponding reduction in peak height (difference 23\%). The observations imply that a single range distribution, measured in the region of dominant $S_{\mathrm{e}}$, will suffice to determine the exponent $q$ with an estimated uncertainty of \pm 0.05 or better. Likewise, if calculations are performed with a fixed value of $q$, like $q$ $=0.5$, i.e., $S_{\mathrm{e}}(v) \propto v$, the results should agree with measured data not only in terms of the mean range but also with respect to the finer details of the range distribution.

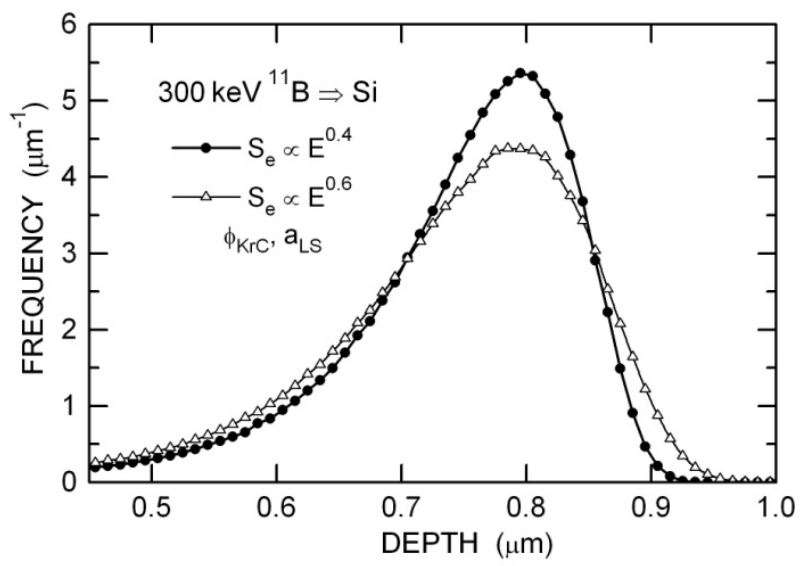

FIG. 6. Comparison of range distributions on $300 \mathrm{keV}$ B in Si calculated for two assumptions concerning the energy dependence of electronic losses. Note the expanded depth scale.

In the region of the Bragg peak, compact analytical formulas for the electronic stopping cross section are not available but they are indispensible here because the procedures used to optimize $S_{\mathrm{e}}$ should be transparent at all energies considered. We have adapted an interpolation formula used by Varelas and Biersack [36] to bridge the gap between the upper limit of velocity-proportional electronic stopping (realistic limit $E^{\downarrow} / M_{1} \approx 10 Z_{1}^{4 / 3} \mathrm{keV} / \mathrm{u}$ ) and the lower limit of validity of Bethe- 
Bloch theory $\left(E^{\uparrow} / M_{1} \approx 400 Z_{1}^{4 / 3} \mathrm{keV} / \mathrm{u}\right)$, i.e., the gap amounts to a factor of about 40 in energy. In essence, the interpolated (fitted) electronic stopping cross section $S_{\mathrm{e}, \mathrm{fit}}$ is derived as $[34,36]$

$$
S_{\mathrm{e}, \mathrm{fit}}=\left(\frac{1}{S_{\mathrm{e}, \mathrm{lo}}}+\frac{1}{S_{\mathrm{e}, \mathrm{hi}}}\right)^{-1}=\frac{S_{\mathrm{e}, \mathrm{lo}} S_{\mathrm{e}, \mathrm{hi}}}{S_{\mathrm{e}, \mathrm{lo}}+S_{\mathrm{e}, \mathrm{hi}}} .
$$

To serve the purpose, $S_{\mathrm{e}, \mathrm{lo}}$ must increase in the gap region with increasing energy, $S_{\mathrm{e}, \mathrm{hi}}$ must increase with decreasing energy. This is easy to accomplish for $S_{\mathrm{e}, \mathrm{lo}}$ by simply allowing the velocity dependence that applies below $E^{\downarrow}$ to remain valid above $E^{\downarrow}$. The tricky part is $S_{\text {e,hi }}$ because, formally, the Bethe-Bloch prediction $S_{\mathrm{e}, \mathrm{BB}}$ passes through a maximum in the gap region (where the prediction for fully stripped projectiles is not valid anyhow [23]). The pre-factor of $S_{\mathrm{e}, \mathrm{BB}}$ and the main component $C_{\mathrm{I}} \beta^{2}$ of the stopping number $L$ in $S_{\mathrm{e}, \mathrm{BB}}[23]$ are retained $\left(C_{\mathrm{I}}=2 \mathrm{~m}_{\mathrm{e}} \mathrm{c}^{2} /\langle I\rangle\right.$, with $\langle I\rangle$ denoting the mean ionization potential of target atoms; $\beta=v / \mathrm{c} ; \mathrm{c}$ is the velocity of light). Going beyond the original interpolation scheme $[34,36], C_{\mathrm{I}} \beta^{2}$ is allowed to be modified by a factor $A_{1}$. The required shape of $S_{\mathrm{e}, \mathrm{hi}}$ is achieved primarily by adding a term $A_{3} / C_{\mathrm{I}} \beta^{2}$, deliberately chosen to be inversely proportional to energy. Another additive term $A_{2}$, independent of energy, provides flexibility for fine adjustment of $S_{\mathrm{e}, \mathrm{hi}}$. Thus

$$
S_{\mathrm{e}, \mathrm{hi}}=B_{1} S_{0} \beta^{-2} Z_{1}^{2} Z_{2} \ln \left(A_{1} C_{\mathrm{I}} \beta^{2}+A_{2}+A_{3} / C_{\mathrm{I}} \beta^{2}\right),
$$

with $S_{0}=4 \pi \mathrm{r}_{0}^{2} \mathrm{~m}_{\mathrm{e}} \mathrm{c}^{2}=5.099 \times 10^{-4} \mathrm{eV} / 10^{15} \mathrm{~cm}^{-2}\left(\mathrm{r}_{0}\right.$ classical electron radius, $\mathrm{m}_{\mathrm{e}}$ electron mass, $B_{1}$ adjustment factor). The original default values for proton stopping were $B_{1}=A_{1}=A_{2}=1, A_{3}=5$ [36]. With $\langle I\rangle=169.5 \mathrm{eV}$ for $\mathrm{Si}[25], Z_{1}=5, Z_{2}=14$, and converting $\beta^{2}$ to reduced energies $E_{\mathrm{vM}}=E / M_{1}$ $(\mathrm{keV} / \mathrm{u})$, we have

$$
\text { - } S_{\mathrm{e}, \mathrm{hi}}(\mathrm{B}, \mathrm{Si})=8.31 \times 10^{4} B_{1} \ln \left(1.295 \times 10^{-2} A_{1} E_{\mathrm{vM}}+A_{2}+77.24 A_{3} / E_{\mathrm{vM}}\right) \mathrm{eV} / 10^{15} \mathrm{~cm}^{-2} \text {. }
$$

To illustrate the fitting procedure we consider the SRIM data for ${ }^{11} \mathrm{~B}-\mathrm{Si}$ again (see Fig. 1). Ignoring the 'undulations' superimposed on the data below $\sim 600 \mathrm{keV}, S_{\mathrm{e}, \text { lo }}$ may be written

$$
S_{\mathrm{e}, \mathrm{lo}}(\mathrm{B}, \mathrm{Si})=k S_{\mathrm{e}, \mathrm{LS}}=13.64 k E_{\mathrm{vM}}^{0.5} \mathrm{eV} / 10^{15} \mathrm{~cm}^{-2} \text {. }
$$

Inserting Eqs. (4a) and (5) in Eq. (3) the factors $B_{1}$ and $A_{\mathrm{i}}(\mathrm{i}=1,2,3)$ were varied until best possible agreement of $S_{\mathrm{e}, \text { fit }}$ with $S_{\mathrm{e}, \text { SRIM }}$ was obtained. The results achieved with $k=1.40, B_{1}=1.03$, $A_{1}=0.95, A_{2}=-1.8$, and $A_{3}=12$ are shown in Fig. 7 as a thick gray line. The fit function agrees quite well with the reference SRIM data (open circles). Note that it was possible to reproduce the non-symmetrical shape of the Bragg peak in the SRIM data which exhibits a steep front end and a 


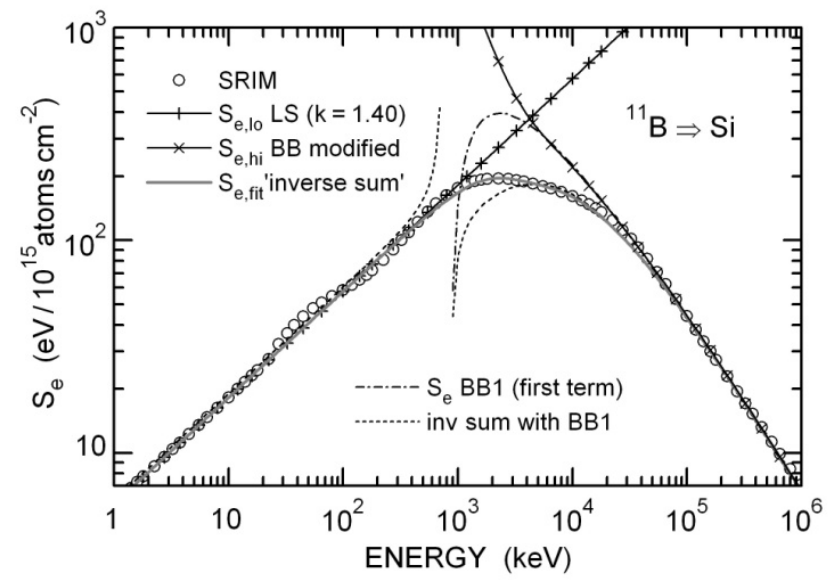

FIG. 7. Illustration of the procedure used to determine an analytical expression for the electronic stopping cross section of B in $\mathrm{Si}$ at energies between $600 \mathrm{keV}$ and $10 \mathrm{MeV}$. The thick gray line was derived as the inverse sum of the functions $S_{\mathrm{e}, \mathrm{lo}}$ and $S_{\mathrm{e}, \mathrm{hi}}$ with the purpose of reproducing the SRIM data shown as open circles. For details of evaluation see text.

comparatively slow fall-off. The steep front end imposed the need for a high value of $A_{3}$. To get the peak position and the fall-off right, $A_{2}$ had to be made negative. For comparison the short-dashed line in Fig. 7, the 'inverse sum with BB1', shows the result of fitting according to Eq. (3) if only the first term of the stopping number is used, i.e., $B_{1}=1$ and $A_{2}=A_{3}=0$ (dash-dotted line). Even though a reasonable fit is obtained for $E>4 \mathrm{MeV}$, the results for $300 \mathrm{keV}<E<4 \mathrm{MeV}$ are useless. Hence, Eq. (4) is required for proper fitting.

\section{RESULTS AND DISCUSSION}

\section{A. Refined data basis}

To determine the basic parameters of nuclear and electronic stopping with high accuracy it was necessary to establish the best possible collection of ranges for B in Si. The data actually used were measured primarily by sputter depth profiling in combination with secondary ion mass spectrometry, SIMS [37,38,39,40,41,42,43,44,45]; results obtained by capacitance-voltage (CV) profilometry $[40,43]$ and nuclear reaction analysis (NRA) [46] were added. Experimental details of the work by the ten different groups are summarized in Table I. In total 98 mean projected ranges $R_{\mathrm{p}}$ reported for ${ }^{11} \mathrm{~B}$ in Si are compiled in Fig. 9 as solid circles. Distributed over almost four orders of magnitude in energy, the data are seen to agree quite well in general. A closer look at the results, however, reveals many significant differences. For example, the SIMS-based ranges of Thevenin et al. [43] reported for $1,1.5,2$, and $2.5 \mathrm{MeV}$ are, on average, $15.5 \pm 2.3 \%$ larger than their counterpart according to 
Svensson et al. [45]. The rather small statistical uncertainty of the mean ratio implies that we are dealing mostly with a systematic difference of unknown origin. One can only speculate that the difference is due to improper calibration of the surface profilometers used to determine the depth of sputtered craters which served to calculate the mean erosion rate (depth calibration).

Table I. Experimental conditions used by the different groups to determine range distributions of B in $\mathrm{Si}$. The impact angle is defined with respect to the surface normal. a-Si: crystalline Si, preamorphized by inert gas or Si bombardment, px-Si: polycrystalline $\mathrm{Si}, \mathrm{x}-\mathrm{Si}$ : crystalline $\mathrm{Si}$ with nonspecified orientation.

\begin{tabular}{|c|c|c|c|c|c|}
\hline Authors & Target & $\begin{array}{l}\text { Mass of B } \\
\text { isotopes }\end{array}$ & $\begin{array}{l}\text { Implantation energy, } \\
\text { impact angle }\end{array}$ & $\begin{array}{l}\text { Method of } \\
\text { analysis }\end{array}$ & $\begin{array}{l}\text { SIMS sputter conditions: } \\
\mathrm{O}_{2} \text { energy, impact angle }\end{array}$ \\
\hline Hofker et al. & $\mathrm{a}-\mathrm{Si}, \mathrm{px}-\mathrm{Si}$ & 11 & $30-800 \mathrm{keV}, 0^{\circ}$ & SIMS & $5.5 \mathrm{keV}, 45^{\circ}$ \\
\hline Wach \& W. & $\mathrm{a}-\mathrm{Si}$ & $10 \& 11$ & $1-40 \mathrm{keV}, 0^{\circ}$ & SIMS & $1.5,2,3 \& 5 \mathrm{keV}, 0^{\circ}$ \\
\hline Wong et al. & $\operatorname{Si}(100)$ & 11 & $1-4 \mathrm{MeV}, 7^{\circ}$ & SIMS & $8 \mathrm{keV}, 37^{\circ}$ \\
\hline Oosterhoff & $\operatorname{Si}(100)$ & 11 & $0.1-1 \mathrm{MeV}, 7^{\circ}$ & SIMS, CV & ?, paid SIMS analysis \\
\hline Svensson et al. 90 & $\operatorname{Si}(111)$ & $10 \& 11$ & $50-250 \mathrm{keV}, 7^{\circ}$ & SIMS & $8 \mathrm{keV}, 37^{\circ}$ \\
\hline Zalm et al. & x-Si, a-Si & $10 \& 11$ & $25-400 \mathrm{keV}, 7^{\circ}$ & SIMS & $3-10.5 \mathrm{keV}, \geq 37^{\circ}$ \\
\hline Thevenin et al. & $\operatorname{Si}(100)$ & 11 & $0.5-3 \mathrm{MeV}, 7^{\circ}$ & SIMS, CV & $10 \mathrm{keV} \mathrm{O}_{2}$ \\
\hline Frey et al. & $\operatorname{Si}(100)$ & 11 & $0.2-7.1 \mathrm{MeV}, 7^{\circ}$ & SIMS & $?,($ IMS-4f) \\
\hline Svensson et al. 93 & $\mathrm{a}-\mathrm{Si}$ & $10 \& 11$ & $0.44-5 \mathrm{MeV}, 0^{\circ}$ & SIMS & $10-12 \mathrm{keV}, 60-70^{\circ}$ \\
\hline Behar et al. & $\mathrm{a}-\mathrm{Si}$ & $10 \& 11$ & $0.05-1 \mathrm{MeV}, 0^{\circ}$ & NRA & n.a. \\
\hline
\end{tabular}

Being unable, in retrospective, to make corrections to the depth calibration of the different sets of range we had to look for ways to minimize the problem. In a first step we have established a fit function $R_{\mathrm{f}}(E)$ representing the raw ranges in Fig. 9 as accurately as possible, over the whole range of energies. Polynomial fit functions to order $s$ serve the purpose but the unequal data density needs to be accounted for. We chose to use the published results for determining mean values of ranges at all energies at which two or more data are available. Selected results $(N=24)$ featuring roughly equal density per $\log E$ interval are shown in Fig. 8 as solid triangles, for ease of identification multiplied by a factor 0.5 . The resulting polynomial fit function $R_{\mathrm{f}}$ to order $s=6$ is shown as a solid line through the raw data (R-square $=0.99995$, standard deviation 0.0084; a fit to order $s=7$ was not used because the energy dependence exhibited unrealistic gradients below $1 \mathrm{keV}$ and above $8 \mathrm{MeV}$ ).

Deviations of the raw $R_{\mathrm{p}}$ data from the fit function are documented as the ratio $r=R_{\mathrm{p}} / R_{\mathrm{f}}$, see Fig. 9(a). The mean is $\langle r\rangle=0.998 \pm 4.7 \%$. The relatively large uncertainty of $4.7 \%$ is attributed to systematic differences between different sets of data, the problem already noted above. To quantify the systematic errors $\delta_{\text {sys }}$, the mean ratio $\rho \equiv\langle r\rangle_{\text {set }}=1 \pm \delta_{\text {syst }}$ was determined separately for each set of 
measurements (owing to SIMS analysis at four different probe energies, see Table I, the data of Wach and Wittmaack [38] represent four sets). Note that, with all data included, $\left\langle\rho N_{\text {set }}\right\rangle=\langle r\rangle\left(N_{\text {set }}\right.$ denotes the number of data per set). The results in Fig. 9(b) may grossly be divided into two groups: 68 data feature small systematic errors, $\delta_{\text {syst }}<3.5 \%$, the remaining 30 data exhibit large deviations between 4.5 and $8.3 \%$. The important point is that even the large systematic errors are almost symmetrically

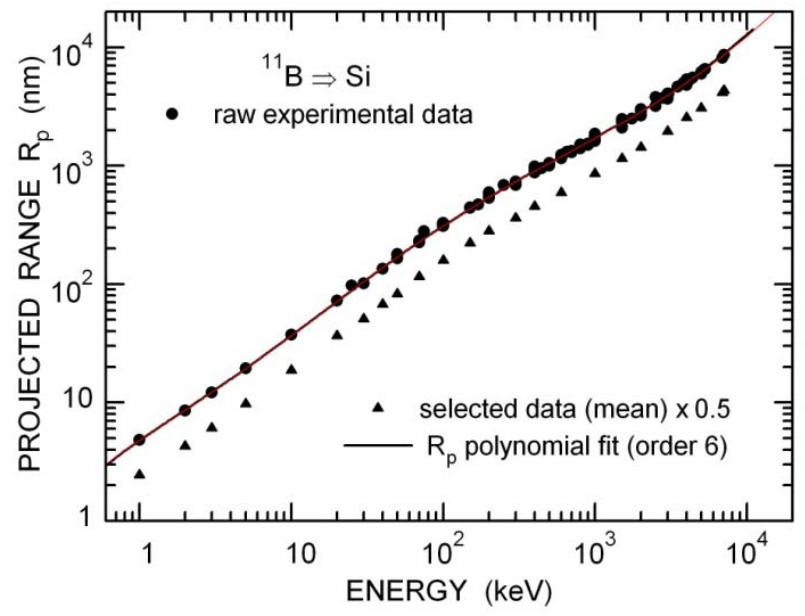

FIG. 8. Compilation of raw and selected mean projected ranges of ${ }^{11} \mathrm{~B}$ in $\mathrm{Si}$ [37-46]. The solid line is a polynomial fit through the selected data. For assignment of the ranges to the various sources see Fig. 9.

distributed around unity, $\left\langle\rho N_{\text {set }}\right\rangle_{\text {large }}=0.993 \pm 5.0 \%$. One should also note the important finding of Fig. 9(b) that the three sets of data by Hofker et al. [37], Wach and Wittmaack [38], and Frey et al. [44] exhibit essentially not systematic error at all $(<0.3 \%)$. Given the fact that, taken together, these three independently derived sets of results cover the whole range of energies, there is good reason to assume that $R_{\mathrm{f}}(E)$ represents the true energy dependence $R_{\mathrm{p}}(E)$ with an uncertainty merely determined by statistical errors of the individual measurements.

Combining the results of Figs. 9(a) and (b), systematic errors in $R_{\mathrm{p}}$ can be removed by determining, separately for each set of data, refined ranges $R_{\mathrm{p}, \mathrm{r}}=R_{\mathrm{p}} / \rho$ and refined ratios $r_{\mathrm{r}}=r / \rho$. The latter should deviate from the mean $\left\langle r_{\mathrm{r}}\right\rangle$ only by statistical errors $\delta_{\text {statis }}$ and, presumably, a few outliers. All results combined are presented in Fig. 9 (c). Statistical analysis showed that 93 data out of a total of 98 can be described quite well by a normal distribution with a standard deviation of only $1.8 \%$ (details not shown). Five data points deviating from $\left\langle r_{\mathrm{r}}\right\rangle=0.999$ by $\left|\delta_{\text {statis }}\right|>5.5 \%$ are encircled in Fig. 9(c). Being located well outside the normal distribution, they are considered outliers. Note that the status 'outlier' is not associated with the magnitude of $\delta_{\text {syst }}$ in the respective study, as one can tell 
from the fact that the SIMS data of Svensson (93) et al. [45], for example, exhibit quite a sizable systematic deviation $\left(\delta_{\text {syst }}=-5.4 \%\right)$ but a rather small statistical uncertainty $\left(\left\langle\delta_{\text {statis }}\right\rangle= \pm 1.8 \%\right)$.

Disregarding the results of Zalm et al. [41] ( $\delta_{\text {syst }}=+8.3 \%,\left\langle\delta_{\text {statis }}\right\rangle= \pm 6.5 \%$, two outliers) and removing the other three outliers, $\left\langle\delta_{\text {statis }}\right\rangle$ ranges from \pm 1.2 to $2.5 \%$ for the different sets of data. Particularly important is the finding that, for each set, $\delta_{\text {statis }}$ does not vary significantly or
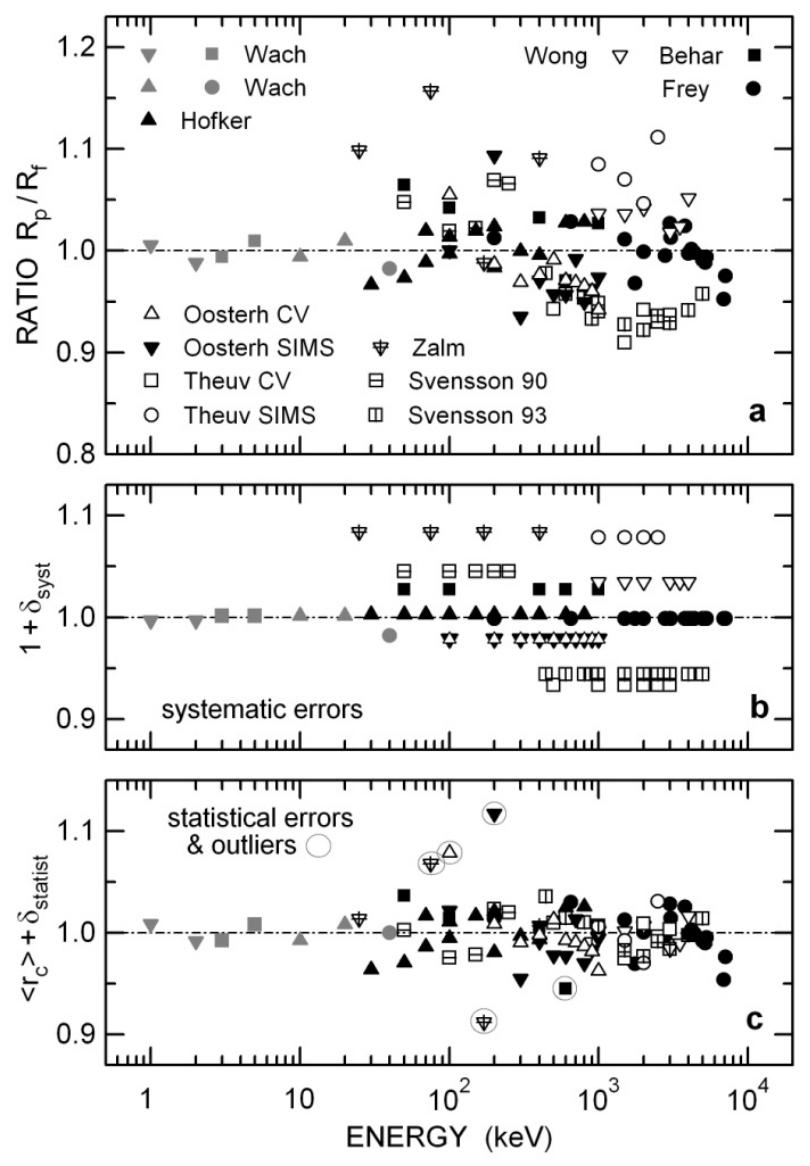

Fig. 9. Ratios $R_{\mathrm{p}} / R_{\mathrm{f}}$ calculated to distinguish between systematic and statistical errors in measured ranges $R_{\mathrm{p}}$. (a) Total scatter of data, (b) systematic errors, and (c) statistical deviations and outliers. For details see text.

systematically with energy, see Fig. 9(c). The absence of a detectable energy dependence of $\delta_{\text {statis }}$ supports the idea that $R_{\mathrm{f}}(E)$ is highly reliable. Quite generally, the results of Fig. 9 confirm the supposition that, with access to a sufficient number of data sets, it is possible to separate statistical uncertainties from systematic errors. A similar, less detailed identification of systematic errors was described very recently by one of us [47] for low-energy, heavy-ion range data based on depth analysis using Rutherford backscattering spectrometry. 


\section{B. Optimum parameters for nuclear interaction and electronic stopping}

To find the input parameters of the Monte Carlo calculations that serve best to accurately reproduce the refined experimental range data of ${ }^{11} \mathrm{~B}$ in $\mathrm{Si}$ one needs to proceed in several steps. Moving from the lowest to the highest energies covered by the data, the first step is to identify the most suitable screening function for the nuclear interaction potential together with an appropriate screening length. The results of such an exercise are presented in Fig. 10. For ease of comparison all data are normalized to the fit function $R_{\mathrm{f}}$. The refined experimental data $R_{\mathrm{p}, \mathrm{r}}$ (open circles) scatter around the line of reference $R_{\mathrm{p}} / R_{\mathrm{f}}=1$. The pronounced dependence of calculated ranges on the chosen screening parameters, already discussed briefly with reference to Fig. 4, is now evident in great detail. The other issue of concern is the dependence of the ranges on $S_{\mathrm{e}}$ through the scaling parameter $k$ of LS electronic stopping. Furthermore, the aim was to confirm (or invalidate) that electronic stopping is proportional to projectile velocity.

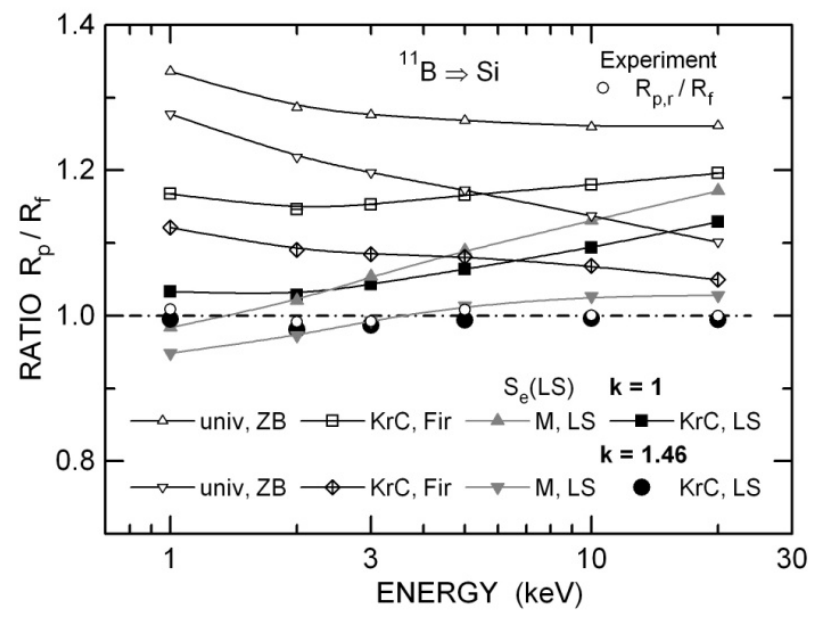

FIG.10. Normalized projected ranges of B in Si calculated with different combinations of screening function and screening length, for $S_{\mathrm{e}}(\mathrm{LS})$ with two different scaling factors $k$. Refined measured ranges $R_{\mathrm{p}, \mathrm{r}}$ (open circles) are shown for comparison. All data are normalized to the fit function $R_{\mathrm{f}}$.

The results of Fig. 10 may be summarized as follows. (i) The ranges of B in Si calculated with $\phi_{\text {univ }}\left(r / a_{\mathrm{ZB}}\right)$ are much too large for $k=1.0$ and significantly too large for $k=1.46$. This implies that ranges derived with TRIMsrim $\left(\phi_{\text {univ }}\left(r / a_{\mathrm{ZB}}\right), k=1.4\right)$ are also too large, exceeding the true value by about $30 \%$ at $1 \mathrm{keV}$ and still by more than $10 \%$ at $20 \mathrm{keV}$. The reason is that the universal potential is too soft, see Fig. 3(a). (ii) Depending on energy, i.e., on the relative contribution of $S_{\mathrm{e}}$ to stopping, the ranges calculated with $\phi_{\mathrm{KrC}}\left(r / a_{\mathrm{Fir}}\right)$ are between 5 and $15 \%$ larger than with $\phi_{\mathrm{KrC}}\left(r / a_{\mathrm{LS}}\right)$. This is due to 
the fact that the screening lengths for $\mathrm{B}$ on Si differ significantly, $a_{\mathrm{LS}}=0.159 \AA, a_{\mathrm{Fir}}=0.142 \AA$, and $a_{\mathrm{ZB}}=0.143 \AA$. Thus, at the same interatomic distance $r$, the absolute value of a selected screening function $\phi(r / a)$ is higher the higher $a$ because the reduced distance is $r / a$ shorter. The important lesson is that the screening length is an input parameter that cannot be chosen deliberately. (iii) The best agreement between experimental and calculated data is obtained with the combination $\phi_{\mathrm{KrC}}\left(r / a_{\mathrm{LS}}\right)$ and $k=1.46$. Admittedly, the uncertainty in determining $k$ is significant at the energies covered in Fig. 10, the estimate being \pm 0.06 at $20 \mathrm{keV}$. However, there is no evidence for a stepwise change in $S_{\mathrm{e}}$ at 11 $\mathrm{keV}\left(0.2 v_{0}\right)$, the issue discussed with reference to Fig. 1. (iv) The ranges calculated with $\phi_{\mathrm{M}}\left(r / a_{\mathrm{LS}}\right) \& k$ $=1.46$ come close to those for $\phi_{\mathrm{KrC}}\left(r / a_{\mathrm{LS}}\right)$ but there is a distinct difference in the energy dependence. The favorable conclusion to be drawn from this finding is that accurate low-energy range data can serve to select the 'right' screening function and screening length for the projectile-target combination under study. (v) For completeness we note that the absolute differences in range for $k=$ 1.0 and 1.46 are very similar in the different screening scenarios, ranging from $0.22 \pm 0.05 \mathrm{~nm}$ at 1 $\mathrm{keV}$ to $10.5 \pm 0.9 \mathrm{~nm}$ at $20 \mathrm{keV}$.

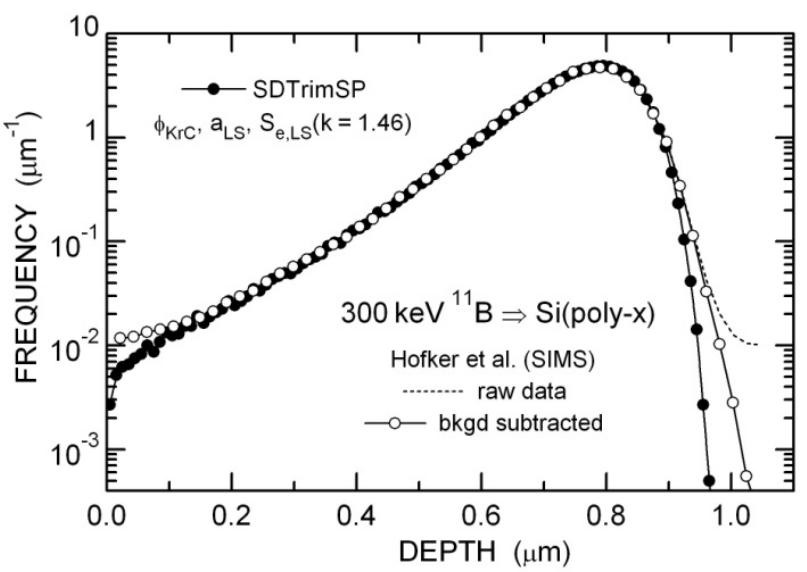

FIG. 11. Comparison of measured and calculated range distributions of $300 \mathrm{keV} \mathrm{B}$ in polycrystalline $\mathrm{Si}$. Background subtraction was applied only at depths exceeding $0.8 \mu \mathrm{m}$.

In order to determine the factor $k$ of LS stopping accurately we compare the shape and position of measured and calculated range distributions, the concept illustrated in Fig. 6. A particularly well suited example is the profile reported by Hofker et al. [37] for impact of $300 \mathrm{keV}{ }^{11} \mathrm{~B}$ on polycrystalline $\mathrm{Si}$ : according to Fig. 9 the ratio $R_{\mathrm{p}} / R_{\mathrm{f}}$ is unity and both, the systematic and the statistical error are undetectably small. Hence, the comparison shown in Fig. 11 did not require any adjustment of the depth calibration. Except for the data in the vicinity of the surface and at the end of 
range the agreement is perfect. This implies that electronic stopping is proportional to the projectile velocity, i.e., LS-type, $S_{\mathrm{e}}=k S_{\mathrm{e}, \mathrm{LS}}$, with a narrowly defined proportionality factor $k=1.46 \pm 0.01$.

The enhanced boron signals observed experimentally near the surface can be attributed to contamination from a variety of sources, an effect studied in some detail [38] after the work of Hofker et al. [37]. The background signal recorded beyond the end of range is most likely due to incomplete suppression of $\mathrm{B}^{+}$ions originating from the walls of the sputtered crater. At the time of the study [37] the concept of electronic gating [48] had not yet been developed so that the authors [37] used less efficient ion optical gating. To derive the 'true' profile we subtracted the background signal, an approach frequently applied to lower the concentration level down to which useful information may be extracted. Compared to the calculated distribution, the background corrected profile exhibits a distinct tail, a well-known feature of sputter depth profiling. With shallow doping profiles, tails are mostly due to bombardment induced mixing [49]. In the case of deep craters exceeding $1 \mu \mathrm{m}$, as in Fig. 11, and notably when produced by oblique ion bombardment (see Table I), one must also consider the likely formation of macroscopically uneven or microscopically rough crater bottoms.

In the context of this study, profile tailing is only of concern with respect to the possible effect on mean projected ranges. Hofker et al. [37] fitted Pearson distributions to their profiles, including the tails. The fitted distributions are very similar to the background corrected profile in Fig. 11 (open circles). Even though the tail looks sizable on a logarithmic scale, the effect on $R_{\mathrm{p}}$ is small, $<0.3 \%$. However, the tails cause errors of increasing magnitude when using Pearson distributions to derive higher moments (straggling, skewness, and kurotosis). In fact, deviations from theory turned out to be progressively larger the higher the moments [37]. On the positive side we note that, in measurements involving crystalline Si targets, Pearson distribution have served well to separate the random component from the channeling tail [39,40,43], see Fig. 2 of ref. 43 for an example.

Whereas the parameters characterizing nuclear interaction and low-velocity electronic stopping were comparatively easy to determine because theoretical concepts are available in analytical form, optimization of $S_{\mathrm{e}}$ in the region of the Bragg peak was more time consuming. Based on the fitting concept described in Sect. IIIB, five variants were tested, two of them only very briefly. The three thoroughly examined versions are presented in Fig. 12. Ratios $r_{\# \mathrm{j}} \equiv R_{\mathrm{p}, \mathrm{r}} / R_{\mathrm{p}, \# \mathrm{j}}$ served to quantify the level of agreement between the 93 refined experimental ranges $R_{\mathrm{p}, \mathrm{r}}$ and the calculated data $R_{\mathrm{p}, \# \mathrm{j}}$. Best agreement was obtained with $S_{\mathrm{e}}$ version \#5, as shown by the solid circles in Fig. 13(a). The fit parameters of Eq. (4a) are: $\mathrm{k}=\mathrm{B}_{1}=1.19, \mathrm{~A}_{1}=0.58, \mathrm{~A}_{2}=0$, and $\mathrm{A}_{3}=2.85$. Averaged over all data 


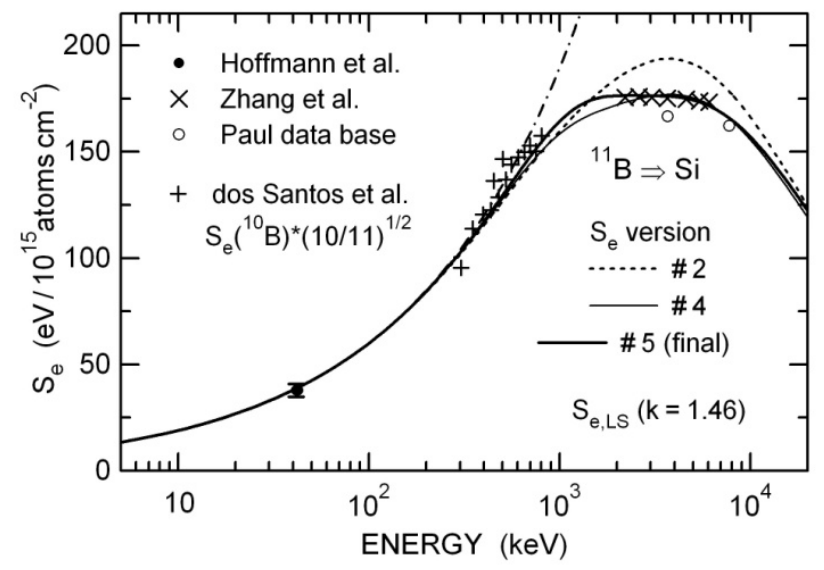

FIG. 12. Versions \# 2, 4, and 5 of the electronic stopping cross section for B in Si, used to calculate ranges for comparison with experimental data. Also shown are the results of a few available experimental studies.
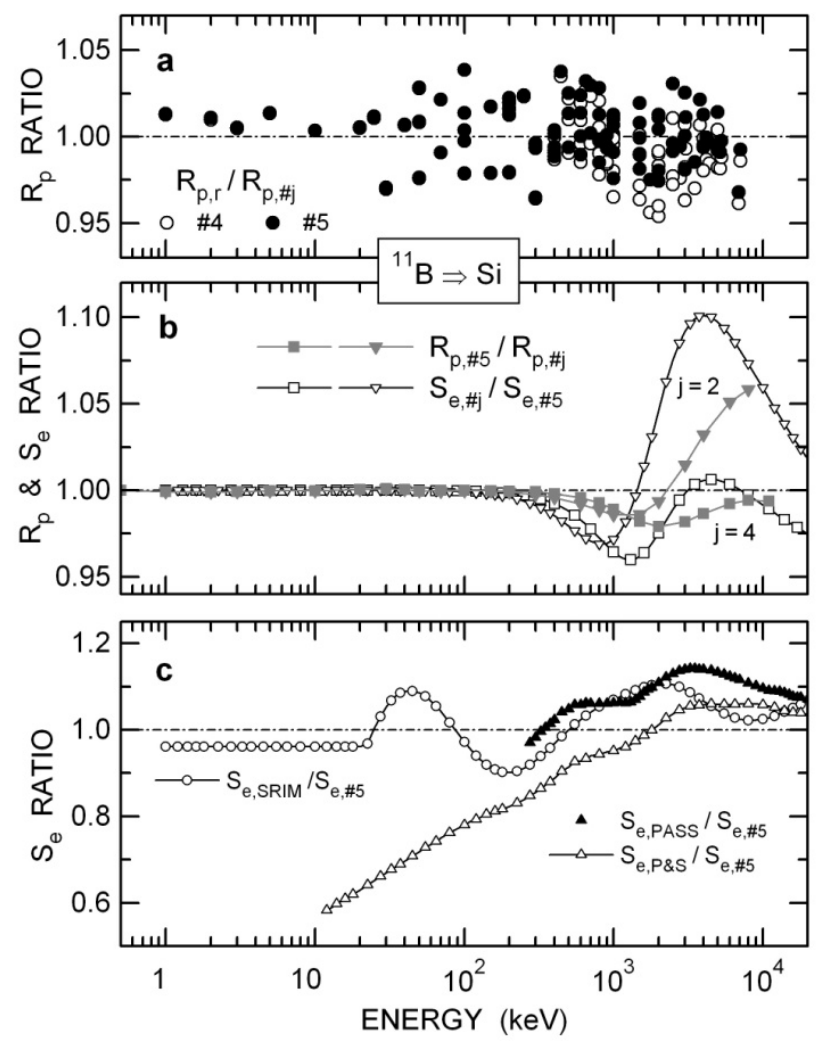

FIG. 13. Ratios of ranges and electronic stopping cross sections. (a) Comparison of experimental and calculated ranges for two versions of $S_{\mathrm{e}}, \# 4$ and \#5. (b) Comparison of calculated ranges, \#5 vs \#2 \& 4, and the corresponding $S_{\mathrm{e}}, \# 2 \& 4$ vs \#5. (c) Comparison of $S_{\mathrm{e}}$ data from empirical libraries

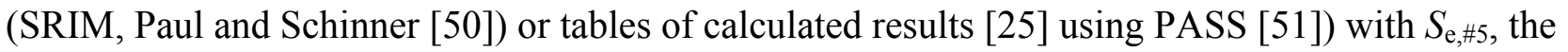
best-fit version of this study. Note that the vertical scale in panel (c) was compressed by a factor four compared to (a) and (b). 
the mean ratio is $\left\langle r_{\# 5}\right\rangle=1.002 \pm 1.7 \%$ (as in the case of $r_{\mathrm{c}}$, Fig. 9(c), the deviations from the mean of $r_{\# 5}$ exhibit a normal distribution). Considering the fact that the ratio comprises data covering almost four orders of magnitude in energy, the agreement may safely be rated excellent.

The sensitivity of calculated ranges to variations of $S_{\mathrm{e}}$ is illustrated in two ways. The open circles in Fig. 13(a) represent $r_{\# 4}$ (open circles that seem to be missing are buried under solid circles located at the same or almost the same position). Evidently $r_{\# 4}$ exhibits a minimum, centered at about $2 \mathrm{MeV}$. Even though the differences between $S_{\mathrm{e}, \# 4}$ and $S_{\mathrm{e}, \# 5}$ are quite small, see Fig. 12, the resulting differences between $R_{\mathrm{p}, \# 4}$ and $R_{\mathrm{p}, \# 5}$ are clearly evident in Fig. 13(a), but only because we managed to assemble a set of range data with small statistical fluctuation. Details of the changes in $R_{\mathrm{p}, \# 5} / R_{\mathrm{p}, \# \mathrm{j}}$ associated with variations of the (inverted) ratios $S_{\mathrm{e}, \# \mathrm{j}} / S_{\mathrm{e}, \# 5}$ are shown in Fig. 13(b). Owing to integration, marked differences in $S_{\mathrm{e}}$ become visible in $R_{\mathrm{p}}$ only at about two times higher energies and the changes in $R_{\mathrm{p}}$ are larger the wider the energy interval over which the changes in $S_{\mathrm{e}}$ extend.

The final version $S_{\mathrm{e}, \# 5}$ may be compared with scarce experimental data from the literature. Of particular interest are the results of Zhang et al. [52] obtained by the standard approach of measuring energy loss in transmission through Si foils, but with up-to-date time of flight spectrometry (uncertainties: energy loss measurements $<1 \%$, foil thickness $\sim 3.5 \%$ [52]). The data (crosses in Fig. 12) fall into the region of the flat-topped Bragg peak and cover a factor 2.8 in energy. The agreement with the present results must be called 'miraculously perfect' (deviations $<1 \%$ ). The same kind of transmission experiment, but with conventional energy loss spectrometry, was used by Hoffmann et al. [53] to determine $S_{\mathrm{e}}$ for $44.2 \mathrm{keV}{ }^{11} \mathrm{~B}$ impact on Si foils. The result, solid circle with $8 \%$ error bar in Fig. 12, is also in very good agreement with $S_{\mathrm{e}, \# 5}$ (difference $<2 \%$ ). We have simulated the experiment with SDTrimSP, assuming a typical $20 \mathrm{~nm}$-foil and a circular acceptance cone of the spectrometer of $0.8^{\circ}$, as specified. The contribution of nuclear stopping to the total energy loss turned out to amount to only 1.2 or $3.7 \%$, depending on whether the most probable or the mean loss is taken into account. These numbers are well below the quoted experimental uncertainty of $8 \%$ [53]. The other literature data in Fig. 12 are also in fair agreement with $S_{\mathrm{e}, \# 5}$. The open circles represent results which, according to ref. 52, are contained in H. Paul's data base [54]. The results reported by dos Santos et al. [55] were derived by backscattering of ${ }^{10} \mathrm{~B}$ from an implanted Bi marker. Considering the various sources of error associated with that concept, the mean deviations of the data (plus signs) from $S_{\mathrm{e}, \# 5}$ are small.

Turning to data tables, Fig. 12(c) shows a comparison with $S_{\mathrm{e}, \# 5}$ in the form of ratios. The

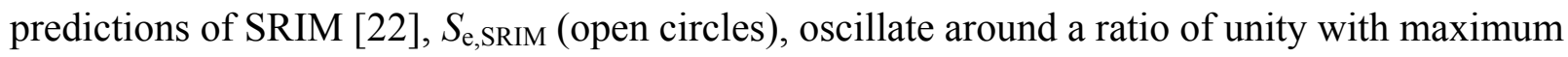


deviations around $\pm 10 \%$. The empirical data contained in the tables of Paul and Schinner [50], $S_{\mathrm{e}, \mathrm{P} \& \mathrm{~S}}$ (open triangles), exhibit very pronounced deviations at low energies, as large as $-40 \%$, already identified in a recent evaluation [23], but above $500 \mathrm{keV}$ the agreement with $S_{\mathrm{e}, \pm 5}$ is generally better than for SRIM. Unexpectedly, the binary-collision code PASS developed by Sigmund and Schinner [51] predicts electronic stopping cross sections $S_{\mathrm{e}, \mathrm{PASS}}$ (solid triangles) [25] that are up to $14 \%$ larger

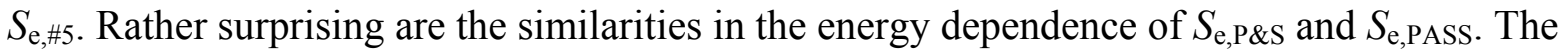
impression is that the author (AS) who contributed to both studies was inspired by ref. 51 when working on ref. 50.

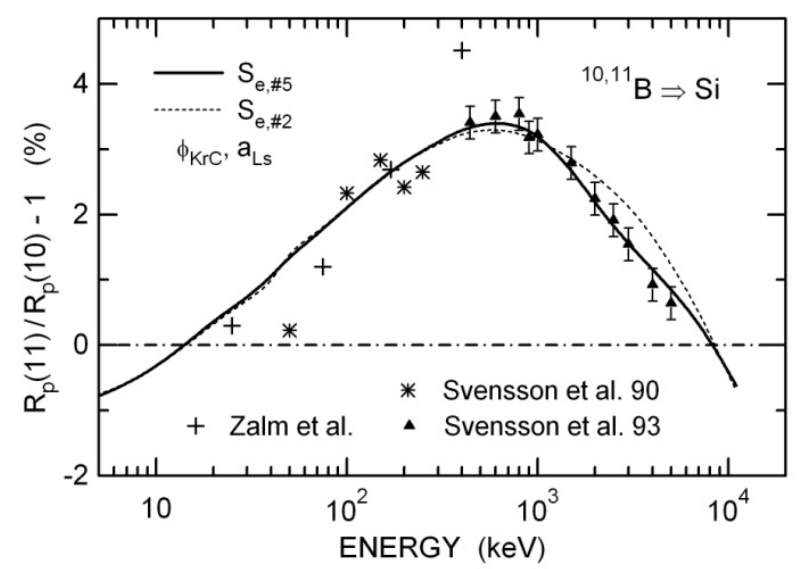

FIG. 14. Isotope effect for ranges of ${ }^{10} \mathrm{~B}$ and ${ }^{11} \mathrm{~B}$ in Si. Results obtained with SDTrimSP using two versions of $S_{\mathrm{e}}$ are compared with experimental data $[41,42,45]$.

To complete comparison of our results with experimental data, Fig. 14 shows measured and calculated isotope effects for ${ }^{10} \mathrm{~B}$ and ${ }^{11} \mathrm{~B}$ in amorphized $\mathrm{Si}$, quantified as $R_{\mathrm{p}}(11) / R_{\mathrm{p}}(10)-1$. The only statistically meaningful set of data is due to Svensson (93) et al. [45]. Systematic errors in range calibration, as documented in Fig. 9(b), cancel out because the profiles of both isotopes were recorded quasi-simultaneously in the same SIMS measurement. The isotope effect calculated using $S_{\text {e,\#5 }}$ agrees with the experimental data (solid triangles) within the uncertainty of $\pm 0.25 \%$ quoted in ref. 45. By contrast, the isotope effect predicted with $S_{\mathrm{e}, \not 2}$ is too high at energies where $S_{\mathrm{e}, \not 2}$ exceeds $S_{\text {e,\#5 }}$ significantly, i.e., between 1 and $10 \mathrm{MeV}$. To summarize, no example was found questioning the reliability of $S_{\mathrm{e}, \# 5 \text {. }}$

\section{CONCLUSIONS AND PERSPECTIVES}

Two remarkable results evolved from this study. First, we have shown that it is possible to determine the parameters quantifying nuclear interaction and electronic stopping by searching for 
best agreement between projected ranges derived from Monte Carlo calculations and data determined experimentally. Second, in order to achieve the highest possible accuracy, it was necessary to remove systematic errors of sizable magnitude that are sometimes buried in measured data reported by different groups. Fortunately, the number of data sets available for this study was large enough so that the systematic errors could be quantified rather safely. The success in search for systematic errors documented here and in a related study [47] should encourage other researchers not to limit discussion of experimental results from different sources to a mere comparison of raw data. Identification of systematic errors is a challenging and somewhat time consuming task. But it is quite satisfying to end up with the finding that data originally showing deviations by 10 or even $20 \%$ may be converted to a refined set with differences less than $2 \%$.

The high accuracy achieved here is rather unique because, for reason of technological interest, ranges of B in Si have been studied by many groups. However, the main goal of this exercise was to show that the concept works well quite generally. Given the fact that the interpolated stopping powers delivered by SRIM [22] at reduced energies $E / M_{1}<1 \mathrm{MeV} / \mathrm{u}$ are often inaccurate or questionable [23], it is highly desirable to establish a significantly improved data basis. The concept outlined above could serve the purpose very well. The aim must be to determine ranges for at least 10-15 projectiles distributed over the whole periodic table, implanted at reduced energies between 0.05 and $200 \mathrm{keV} / \mathrm{u}$ so that the ranges very roughly extend from $10 \mathrm{~nm}$ to $10 \mu \mathrm{m}$. The method of choice for measuring ranges is SIMS. Suitable targets are $\mathrm{Si}$ and Ge because, under well chosen sputter conditions, they retain a reasonably smooth surface during analysis. For good accuracy it is advisable to select projectiles exhibiting appropriate yields, either as positive or as negative ions. Elements known to exhibit redistribution artifacts in SIMS should be excluded as projectiles. Shallow range distributions must be measured at low probe energies.

With range data thus obtained one should be able to clarify several issues of concern. In the region of dominant nuclear stopping, for example, the question is whether a preferable screening function will emerge, valid across the periodic table and including the definition of a reliable $Z_{1,2}$ dependence of the screening length. As to electronic stopping, it will be of interest to see which similarities and differences exist in the energy (velocity) and $Z_{1}$ dependence of $S_{\mathrm{e}}$. Depending on the results it may be possible to establish much improved interpolation schemes for nuclear and electronic stopping cross sections. 
[1] S. N. Markin, D. Primetzhofer, S. Prusa, M. Brunmayr, G. Kowarik, F. Aumayr, and P. Bauer, Phys. Rev. B 78, 195122 (2008).

[2] K. Wittmaack, Phys. Rev. B 91, 115434 (2015).

[3] P. Riccardi, R. Baragiola, and C. A. Duke, Phys. Rev. B 92, 045425 (2015).

[4] S. N. Markin, D. Primetzhofer, M. Spitz, and P. Bauer. Phys. Rev. B 80, 205105 (2009).

[5] E. D. Cantero, G. H. Lantschner, J. C. Eckardt, and N. R. Arista, Phys. Rev. A 80, 032904 (2009).

[6] D. Goebl, D. Roth, and P. Bauer, Phys. Rev. A 87, 062903 (2013).

[7] C. Celedón, E. A. Sánchez, M. S. Moreno, N. R. Arista, J. D. Uribe, M. Mery, J. E. Valdés, and P. Vargas, Phys. Rev. A 88, 012903 (2013).

[8] D. Primetzhofer, S. Rund, D. Roth, D. Goebl, and P. Bauer, Phys. Rev. Lett. 107, 163201 (2011).

[9] D. Primetzhofer, S. N. Markin, D. V. Efrosinin, E. Steinbauer, R. Andrzejewski, and P. Bauer, Nucl. Instrum. Methods Phys. Res., Sect. B 269, 1292 (2011).

[10] P. Sigmund, Eur. Phys. J. D 47, 45 (2008).

[11] Y. Zhang, I.-T. Bae, K. Sun, C. Wang, M. Ishimaru, Z. Zhu, W. Jiang, and W. J. Weber, J. Appl. Phys. 105, 104901 (2009).

[12] C. Lan, J. M. Xue, Y. Zhang, J. R. Morris, Z. Zhu, Y. Gao, Y. G. Wang, S. Yan, and W. J. Weber, Nucl. Instrum. Methods Phys. Res., Sect. B 286, 45 (2012).

[13] K. Jin, Y. Zhang, Z. Zhu, D. A. Grove, H. Xue, J. Xue, and W. J. Weber, J. Appl. Phys. 115, 044903 (2014).

[14] D. Cai, N. Grønbach-Jensen, C. M. Snell, and K. M. Beardmore, Phys. Rev. B 54, 17147 (1996).

[15] Y. Zhang, G. Possnert, and W. J. Weber, Appl. Phys. Lett. 80, 4662 (2002).

[16] E. D. Cantero, R. C. Fadanelli, C. C. Montanari, M. Behar, J. C. Eckardt, G. H. Lantschner, J. E. Miraglia, and N. R. Arista, Phys. Rev. A 79, 042904 (2009).

[17] P. Sigmund and A. Schinner, Nucl. Instrum. Methods Phys. Res., Sect. B 382, 15 (2016).

[18] J. H. Ormrod and H. E. Duckworth, Can. J. Phys. 41, 1424 (1963).

[19] P. Hvelplund and B. Fastrup, Phys. Rev. 165, 408 (1968).

[20] P. Hvelplund, Mat. Fys. Medd. Dan. Vid. Selsk. 38, 1 (1971).

[21] L. G. Glazov and P. Sigmund, Nucl. Instrum. Methods Phys. Res., Sect. B 207, 240 (2003).

[22] J. F. Ziegler, M. D. Ziegler, and J. P. Biersack, The Stopping and Range of Ions in Matter, $<$ www.srim.org $>$

[23] K. Wittmaack, Nucl. Instrum. Methods Phys. Res., Sect. B 380, 57 (2016).

[24] J. Lindhard and M. Scharff, Phys. Rev. 124, 128 (1961).

[25] ICRU Report No. 73, Stopping of Ions Heavier than Helium, J. ICRU 5, No. 1 (2005).

[26] W. D. Wilson, L. G. Haggmark, and J. P. Biersack, Phys. Rev. B 15, 2458 (1977).

[27] W. Wach and K. Wittmaack, Phys. Rev. B 27, 3528 (1983).

[28] H. E. Schiøtt, Radiat. Eff. 6, 107 (1970).

[29] G. Molière, Z. Naturforsch. 2a, 133 (1947).

[30] J. F. Ziegler, J. P. Biersack, and U. Littmark, The Stopping and Range of Ions in Solids (Pergamon, New York, 1985).

[31] O. B. Firsov, Zh. Eksp. Teor. Fiz. [Sov. Phys.-JETP] 32, 1464 (1957).

[32] S. Kalbitzer and H. Oetzmann, Phys. Lett. 59A, 197 (1976). 
[33] A. Mutzke, R. Schneider, W. Eckstein, and R. Dohmen, Max-Planck-Institut für Plasmaphysik, IPPReport 12/8 (2011).

[34] J. P. Biersack and L. G. Haggmark, Nucl. Instrum. Methods 174, 257 (1980).

[35] K. Wittmaack and A. Mutzke, Nucl. Instrum. Methods Phys. Res., Sect. B 281, 37 (2012).

[36] C. Varelas and J. Biersack, Nucl. Instrum. Methods 79, 213 (1970).

[37] W. K. Hofker, D. P. Oosthoek, N. J. Koeman, and H. A. M. de Grefte, Radiat. Eff. 24, 223 (1975).

[38] W. Wach and K. Wittmaack, Nucl. Instrum. Methods 194, 113 (1982).

[39] H. Wong, E. Deng, N. W. Cheung, P. K. Chu, E. M. Strathman, and M. D. Strathman, Nucl. Instrum. Methods Phys. Res., Sect. B 21, 447 (1987).

[40] S. Oosterhoff, Nucl. Instrum. Methods Phys. Res., Sect. B 30, 1 (1988).

[41] P. C. Zalm, G. M. Fontijn, K. T. F. Janssen, and C. J. Vriezema, Nucl. Instrum. Methods Phys. Res., Sect. B 42, 397 (1989).

[42] B. G. Svensson, J. T. Linnros, and G. Holmèn, J. Appl. Phys. 68, 73 (1990).

[43] P. Thevenin, J. J. Grob, R. Stuck, and P. Siffert, Nucl. Instrum. Methods Phys. Res., Sect. B 62, 346 (1992).

[44] L. Frey, S. Bogen, L. Gong, W. Jung, H. Ryssel, and J. Gyulai, Nucl. Instrum. Methods Phys. Res., Sect. B 62, 410 (1992).

[45] B. G. Svensson, M. C. Ridgway, and M. Petravić, J. Appl. Phys. 73, 4836 (1993).

[46] M. Behar, M. Weiser, S. Kalbitzer, D. Fink, and F. L. Grande, Nucl. Instrum. Methods Phys. Res., Sect. B 34, 316 (1988).

[47] K. Wittmaack, Nucl. Instrum. Methods Phys. Res., Sect. B 388, 15 (2016).

[48] K. Wittmaack, Appl. Phys. 12, 149 (1977).

[49] K. Wittmaack, Vacuum 34, 119 (1984).

[50] H. Paul and A. Schinner, At. Data Nucl. Data Tables 85, 377 (2003).

[51] P. Sigmund and A. Schinner, Eur. Phys. J. D 12, 425 (2000).

[52] Y. Zhang, W. J. Weber, and H. J. Whitlow, Nucl. Instrum. Methods Phys. Res., Sect. B 215, 48 (2004).

[53] I. Hoffmann, E. Jäger, and U. Müller-Jahreis, Radiat. Eff. 31, 57 (1976).

[54] Stopping Power of Matter for Ions, IAEA Nuclear Data Services, https://www-nds.iaea.org/stopping/

[55] J. H. R. dos Santos, M. Behar, P. L. Grande, H. Boudinov, R. Stoll, Chr. Klatt, and S. Kalbitzer, Phys. Rev. B 55, 13651 (1997). 